\title{
Um modelo dinâmico considerando uma estratégia de desenvolvimento de catching-up tecnológico
}

\author{
A dynamic model considering the development strategy of technological \\ catching-up
}

Luis Eduardo Esteves*

\begin{abstract}
Resumo: O objetivo do trabalho é discutir o papel do processo de catching-up no crescimento econômico e oferecer um modelo geral que incorpore as principais contribuições sobre esse tema. Um aspecto chave no catching-up é a necessidade de o país atrasado realizar esforços para reduzir a distância com relação à fronteira tecnológica. O ponto de partida é o argumento de Narula (2004), que relaciona a capacidade absortiva com o grau de desenvolvimento do país. Com o objetivo de endogenizar tanto a capacidade absortiva quanto o hiato tecnológico, desenvolve-se um modelo baseado nas premissas de Verspagen (1993), em que a relação entre o hiato tecnológico e a capacidade absortiva é não-linear. Como sugerido por esse autor, o hiato representa uma oportunidade de imitação, mas também um maior desafio competitivo. Os resultados ressaltam a necessidade de existência de uma capacidade absortiva mínima como condição de estabilidade para um sistema de economias abertas com diferentes níveis de avanço tecnológico.
\end{abstract}

Palavras-chave: Catching-up; Hiato tecnológico; Capacidade absortiva

\begin{abstract}
The objective of this paper is to discuss the role of the process of catching-up in economic growth. Additionally, it also aims to incorporate in a model various contributions on the dynamics of the catching up. A key aspect in the process of catchingup are the efforts of the laggard economy to absorb foreign technology. We take as a point of departure the argument of Narula (2004) that relates the absorptive capacity with the degree of development of the country. Based on Verspagen (1993), we assume that the relation between the technological gap and the absorptive capacity is non-linear. As suggested by the author, the gap represents an opportunity of imitation, but also a bigger competitive challenge. It is shown that there exists a minimum degree of domestic absorptive capabilities necessary for having stability in an international system formed by open economies with different degrees of technological capabilities.
\end{abstract}

Keywords: Catching-up; Technological gap; Absorptive capacity

JEL Code: 043

*Especialista em Regulação da ANP. Mestre em Desenvolvimento Econômico pela UFPR. Candidato ao PhD na Université Grenoble-Alpes. E-mail: lesteves@anp.gov.br. 


\section{Introdução}

O objetivo deste trabalho é discutir o processo de catching-up tecnológico considerando como marco teórico um modelo matemático. Adicionalmente, esse trabalho busca oferecer um modelo geral em que as distintas perspectivas sobre esse processo sejam apresentadas de maneira formal.

Uma parte da literatura tem se dedicado a analisar o potencial das economias conhecidas como tecnologicamente atrasadas. Adotando-se uma perspectiva macro, o crescimento econômico é garantido por meio de um processo de catching-up que resulta, ao final desse, em convergência da renda per capita entre os países. Porém, a condição necessária para o início do processo de catching-up e, portanto, de convergência é baseada em uma capacidade dos países atrasados explorarem os conhecimentos já consolidados pelos países líderes. Assim sendo, os países tecnologicamente atrasados podem reduzir o diferencial de renda por meio da introdução de novas técnicas que não estão na fronteira do conhecimento. Sinteticamente, a principal hipótese sobre o conceito de catchingup é a possibilidade de o país tecnologicamente atrasado crescer a taxas maiores que os países centrais, localizados na fronteira da tecnologia mundial (ABRAMOVITZ, 1986; UNCTAD, 2005).

A literatura frequentemente cita exemplos bem-sucedidos do processo catching-up tais como o Japão e a Coréia do Sul. Porém, observando o processo de desenvolvimento dos países integrantes da OCDE, a maioria desses países também pode ser classificada como um relevante exemplo de um processo de catching-up. Entretanto, fora do conjunto da OCDE, os países têm obtido desempenhos menos marcantes. Em geral, podemos afirmar que um pequeno grupo de países obteve um alto crescimento enquanto outro grupo representando a maior parte dos países não atingiram essa trajetória de convergência (FAGERBERG, 1988b; 1994).

Segundo Abramovitz (1986), Baumol (1986) e Maddison (1987) existe evidência empírica revelando uma convergência da renda per capita entre os países. Tais resultados são criticados por De Long (1988), baseando sua crítica na amostra que evidencia um viés de seleção realizado ex-post. Uma vez que o conjunto da amostra do primeiro estudo baseia-se nos países da OCDE, esta crítica é justificada. 
Como resposta a essa crítica, o estudo de Baumol, Blackman e Wolff(1989) estende a amostra para todos os países com disponibilidade de dados para o período analisado. $\mathrm{O}$ estudo demonstra que, embora o grupo que obtém a convergência, i.e., iniciam a trajetória do catching-up, se encontre dentro da área da OCDE, existem poucas evidências que suportem a hipótese de convergência entre todos os países incluídos na análise. Na tentativa de explorar mais os dados, os autores inserem mais uma variável explicativa, o esforço da nação dedicado a massificação da educação, sendo utilizado como proxy os anos de estudo dos indivíduos. Com esta nova variável explicativa, observa-se que a convergência é influenciada por esse esforço. Em resumo, o processo de catching-up não ocorre pelo simples fato de existir o hiato tecnológico, sendo necessária a existência de um esforço do país para iniciar uma trajetória de convergência da renda.

Em outro estudo, Targetti e Foti (1997) realizam testes empíricos para elucidar as diferenças existentes entre os dois distintos grupos de países tendo como principal variável explicativa o hiato tecnológico. Assim sendo, desenvolvem um modelo baseado em duas hipóteses. A primeira é a existência do processo de catching-up. A segunda é sobre o crescimento cumulativo. Para tanto, os autores tomam como amostra 9 países da OCDE, 9 países Latino Americanos e 7 países do Leste Asiático para o período entre 1950-88. Uma das conclusões é a relação positiva entre o tamanho do hiato tecnológico e a produtividade do fator trabalho. Portanto, quanto maior for o hiato tecnológico, maior será a tendência para que haja um crescimento da produtividade dos países tecnologicamente atrasados. Nesse sentido, existe uma tendência de convergência da renda de todos os grupos de países selecionados. Todavia, quando analisados grupo a grupo, a convergência da renda ocorre somente entre os países da OCDE e do Leste Asiático correspondendo aos países da América Latina uma divergência da renda (TARGETTI; FOTI, 1997).

Nesse contexto, a explicação para o sucesso ou fracasso na convergência da renda e, portanto, de sua trajetória de crescimento é determinada pelo grau de internalização da tecnologia. Adicionalmente, o elemento mais relevante para o processo de internalização é o grau de esforço do país. Consequentemente esses dois elementos, o grau de esforço e o processo de acumulação de tecnologia são elementos fundamentais para determinar a convergência da renda dos países. 
Entretanto, o princípio de que existe um custo para a internalização de tecnologias representa uma alternativa à tradição neoclássica, principalmente fundamentada em modelos desenvolvidos por Solow a partir da década de 50 . Esses modelos supõem que a tecnologia é um bem público e, portanto, não existem custos associados à sua aquisição ou mesmo difusão. Empiricamente, estudos baseados nessa hipótese pouco explicam acerca da divergência da renda entre os diferentes países (FAGERBERG, 1988b; 1994).

Dada tal disparidade entre a teoria e a evidência empírica, vários autores têm se concentrado em teorias alternativas à tradição neoclássica, focando seus esforços na explicação das divergências entre o nível de renda per capita. Dentro deste segmento, grande parte dos autores têm empregado esforços para incrementar a compreensão quanto ao papel desempenhado pelo hiato tecnológico e ao esforço do país para acumular tecnologia. Esses trabalhos tem se baseado em dois métodos. O primeiro, parte de um estudo histórico demonstrando a evolução dos países no processo de catching-up tecnológico a partir de uma perspectiva de longo prazo levando-se em consideração mais fatores relevantes do que um modelo matemático ou estatístico é capaz de analisar. O segundo trata de trabalhos empíricos baseados em modelos econométricos. Ambas metodologias têm providenciado suporte para essa visão alternativa que introduz custos para a acumulação da tecnologia e subsequente convergência da renda entre os países (FAGERBERG, 1988b; 1994).

Concluímos que os atuais modelos de catching-up representados unicamente pelo hiato tecnológico não podem capturar toda a complexidade do processo, muito menos revelar particularidades desse processo dinâmico. A solução proposta por esse trabalho objetiva introduzir uma segunda variável para observar a sua dinâmica dentro desse modelo. Neste contexto, o trabalho propõe internalizar o esforço do país para o seu desenvolvimento com o objetivo de melhorar a compreensão do processo de catching-up (NARULA, 2004; COHEN; LEVINTHAL, 1989; CRISCUOLO; NARULA, 2002).

Porém, esse esforço do país para acumular tecnologias e reduzir o hiato da renda incorpora um conjunto de elementos de difícil mensuração. Esse conjunto de elementos é denominado por Abramovitz (1986) de capacidade social, delimitando esse conjunto por meio de duas grandes categorias. A primeira 
incorpora as atitudes sociais e as instituições políticas, englobando o empenho e a forma de tratamento do desenvolvimento tecnológico. A segunda conta com os elementos que determinam a habilidade dos países para absorver de forma eficiente a tecnologia já existente. Esse último grupo, a literatura tem chamado de capacidade absortiva, definida por Dahlman e Nelson (1995) como: "the ability to learn and implement the technologies and associated practices of already developed countries". Essa ideia é aplicada por Cohen e Levinthal (1989) ao desenvolverem o conceito para as firmas, ou seja, aplicando o conceito à esfera micro.

Seguindo à iniciativa de Cohen e Levinthal (1989), a literatura desenvolveu e aplicou o conceito de capacidade absortiva na análise das interações da firma. Até o presente momento, poucos estudos se dedicaram a aplicar o conceito de capacidade absortiva no nível macro, criando uma janela de oportunidade para o emprego desse conceito com o foco na compreensão do processo de acumulação de conhecimentos para os países (CRISCUOLO; NARULA, 2002).

Um dos pioneiros em condicionar o crescimento econômico à capacidade absortiva de cada país é Verspagen (1993). Nesse estudo pioneiro, o autor relaciona a restrição externa ao crescimento com a necessidade de absorção de tecnologias, criando um modelo de crescimento restringido tanto pela elasticidade renda das exportações e das importações ${ }^{1}$, quanto pelo hiato tecnológico. Porém é importante ressaltar que o hiato tecnológico influencia o crescimento a partir das funções de demanda por importações e exportações, agindo como uma mensuração da qualidade dos produtos. Por fim, o hiato tecnológico e, consequentemente a qualidade dos produtos exportados e importados, é influenciado pela capacidade absortiva do país. Nesse contexto, a capacidade absortiva do país tem um papel central na análise da trajetória de crescimento da nação.

\footnotetext{
${ }^{1}$ Esta relação é conhecida como Lei de Thirlwall. Essa lei declara que a taxa de crescimento do país é determinada, em longo prazo, pela balança de pagamentos, i.e., pela disponibilidade de divisas externas. Em outros termos nenhum país, em longo prazo, pode crescer acumulando saldos negativos no balanço de pagamentos. Em algum momento haverá, uma restrição de divisas internacionais que provocará uma contração no crescimento, impondo ao país uma taxa de crescimento condizente com o equilíbrio da balança de pagamentos, sumarizado pela lei e pela balança comercial. Assim, a lei sumariza essa restrição ao crescimento de longo prazo pela razão entre as elasticidades renda das exportações multiplicada pela taxa de crescimento do resto do mundo dividido pela elasticidade renda das importações. Portanto, quanto maior for a elasticidade renda das exportações, maior será a taxa de crescimento compatível com o equilíbrio na balança de pagamentos. O pensamento inverso vale para a elasticidade renda das importações. Para maiores detalhes veja: McCombie e Thirlwall (1994).
} 
Entretanto, no estudo de Verspagen (1993), a capacidade absortiva é determinada por parâmetros exógenos ao modelo, o que não condiz com a realidade observada por Narula (2004). Para esse último autor a capacidade absortiva do país é um fator determinante do hiato tecnológico e, consequentemente, essencial para revelar a dinâmica do crescimento econômico.

Neste contexto esse trabalho tem como objetivo internalizar a capacidade absortiva à um modelo dinâmico considerando o hiato tecnológico existente entre países desenvolvidos e dos tecnologicamente atrasados. Enquanto o primeiro grupo de países se encontra na fronteira tecnológica, o segundo conjunto demanda um esforço interno para gerar uma capacidade absortiva. Portanto, o processo de catching-up demanda o esforço de desenvolvimento de capacidade absortiva (NARULA, 2004; CRISCUOLO; NARULA, 2002; COHEN; LEVINTHAL, 1989; UNCTAD, 2005; NELSON; WINTER, 1982; FREEMAN, 1995).

Nesse modelo buscamos internalizar uma perspectiva de co-evolução entre capacidade de absorção e hiato que foi inicialmente desenvolvida por Narula (2004). A partir dessa perspectiva, há uma relação não linear entre a habilidade de um país absorver conhecimentos externos com o seu estágio de desenvolvimento tecnológico. Assim, conforme o país acumula tecnologias e se aproxima da fronteira tecnológica, aumenta a dificuldade de absorção, demonstrando que a acumulação de tecnológicas também dificulta a absorção de tecnologias adicionais. Associamos essa co-evolução para complementar o trabalho de Verspagen (1993), representando certa fadiga do modelo de cathing-up provocado pelo acumulo de conhecimento quando os países se aproximam da fronteira tecnológica.

Para tanto, dividimos o trabalho em cinco seções além desta introdução e da conclusão. Na primeira seção, analisaremos as principais contribuições da literatura sobre o processo de cathing-up, ressaltando a convergência que estas apresentam, gerando fatos estilizados que serão, oportunamente, aproveitados no desenvolvimento das equações que caracterizarão o modelo dinâmico que será apresentado como resultado da pesquisa.

A segunda seção é dedicada ao hiato tecnológico, que reflete os fatos estilizados já retratados na primeira seção. Nesta seção já obtemos a primeira equação do modelo dinâmico. 
O tema da terceira seção é a capacidade absortiva. Aqui consideramos as contribuições da literatura para desenvolver a equação relacionada ao comportamento da capacidade absortiva.

Dedicamos a quarta seção à solução do modelo dinâmico que agrega as duas equações desenvolvidas nas seções dois e três. Nesta seção obteremos a trajetória que é desenvolvida pelos países durante o processo de catching-up tecnológico.

A quinta seção expõe uma análise gráfica da solução encontrada para o modelo dinâmico. Esta seção é particularmente útil para visualizar os resultados que emergem desse modelo.

\section{Contribuições da literatura}

O objetivo dessa seção é introduzir as principais contribuições da literatura acerca do processo de catching-up, bem como agrupar as contribuições de forma a demonstrar a convergência das premissas. Assim, ao final da seção traremos o rol de fatos estilizados que serão utilizados como embasamento para o modelo desenvolvido mais adiante. A partir da revisão da literatura, assumimos duas premissas para o modelo. Em primeiro lugar, o país tecnologicamente atrasado necessita de uma capacidade absortiva mínima para internalizar as tecnologias disponíveis e iniciar o processo de catching-up. Esse fato revela a necessidade do país atrasado realizar um esforço inicial para iniciar o processo de desenvolvimento através do catching-up. Em segundo lugar, o processo de aquisição de capacidades é cumulativo, de modo que conhecimentos já internalizados impulsionem a aquisição de mais capacidades, implicando na internalização de novos conhecimentos. Entretanto, o custo de absorção aumenta conforme o hiato tecnológico se reduz, demonstrando a dificuldade de absorver tecnologias que se encontram na fronteira do conhecimento.

Conforme ressaltado por Abramovitz (1986), as capacidades sociais de cada país devem ser suficientemente desenvolvidas para que o país explore o potencial dado pelo hiato tecnológico. Em linha com este autor, Cohen e Levinthal (1989) ressaltam a necessidade de aquisição da habilidade de "learning to learn", ou seja, a necessidade de que as pessoas aprendam a lidar com as mudanças tecnológicas proporcionadas pelo avanço da ciência para que o conhecimento seja internalizado. O estudo da UNCTAD (2005) afirma que os países menos desenvolvidos ainda 
não têm um nível mínimo de capacidade absortiva, de modo a capacitá-los para o início do processo de crescimento baseado no catching-up.

Outro marco teórico é fornecido por Nelson e Phelps (1966) ao afirmarem que a educação é fundamental para que os indivíduos possam se adaptar a mudanças proporcionadas pelo progresso tecnológico. Adicionalmente, os autores desenvolvem um modelo no qual a difusão da tecnologia segue um índice médio do grau de educação da população. Portanto, quanto maior for o grau de educação das pessoas, maior será a velocidade de propagação da tecnologia, demonstrando que o esforço do país ao elevar a educação da população influencia positivamente a taxa de propagação/internalização das tecnologias.

Outra visão sobre o processo é desenvolvida por Fagerberg (1994). O autor destaca que o hiato tecnológico deve ser visualizado como uma promessa de crescimento, caso o país atinja um nível mínimo para absorver as tecnologias. Neste contexto, o autor enfatiza que o crescimento por meio do hiato tecnológico não é automático, dependendo fundamentalmente das capacidades sociais do país.

Em seu trabalho, Verspagen (1993) afirma que o progresso tecnológico gera transbordamentos, entretanto, nem todos os transbordamentos podem ser capturados pela sociedade. Neste sentido, quanto maior for a capacidade de aprendizado da sociedade, maiores serão os transbordamentos capturados.

Finalmente, Narula (2004) cria o conceito de uma capacidade absortiva mínima que é necessária para dar impulso ao processo de catching-up.

Nesse sentido, é consenso entre os autores que existe uma necessidade de desenvolvimento de capacidades para que o país tecnologicamente atrasado possa iniciar o processo de catching-up. Esse esforço deve ser realizado e, efetivamente, gerará algum custo para o seu respectivo desenvolvimento. Esse custo é representado pelos esforços realizados no país para que a população aumente a sua educação ou pelas políticas governamentais que são implementadas objetivando impulsionar as conexões realizadas dentro dos centros difusores das novas tecnologias (empresas, universidades, centros de pesquisa, etc.), i.e., dentro do Sistema Nacional de Inovação (SNI).

Segundo Cohen e Levinthal (1989), a estrutura cognitiva é cumulativa em relação a capacidade absortiva. Adicionalmente há um custo na absorção de 
informações ou tecnologias que é tanto maior quanto mais complexo for o conhecimento.

Ainda nessa direção, o estudo da UNCTAD (2005) considera que há a possibilidade de criar-se um círculo virtuoso onde o crescimento do conjunto de conhecimentos permitiria uma maior difusão das tecnologias. Assim, o desenvolvimento tecnológico dos países na fronteira tecnológica também tende a reduzir o custo da transferência tecnológica no curto prazo.

Nessa linha Fagerberg (1994) propõe que a cumulatividade é uma característica presente no processo de introdução das novas tecnologias. Não obstante, o autor enfatiza que existem custos em sua internalização.

Ainda sobre a cumulatividade, Verspagen (1993) declara que em um processo do catching-up, ela é definida pelo tamanho do hiato tecnológico. Neste contexto, existe tanto um fator positivo (impulsionador) quanto um fator negativo (contracionista). O fator positivo é gerado pelo acúmulo de conhecimentos que influência diretamente a elevação das capacidades de cada país. Já o fator negativo é proporcionado pela elevação na dificuldade de imitar tecnologias mais próximas à fronteira tecnológica.

Finalmente, Narula (2005) considera que a capacidade de cada país depende do seu estágio de desenvolvimento. Consequentemente, há uma característica de cumulatividade no início do processo (desde que ultrapassado um nível mínimo de capacidade absortiva), mas que declina conforme o processo de desenvolvimento encontra um limite.

Consequentemente, assumimos que o processo catching-up é cumulativo e dependente de um esforço do país. Esse processo cumulativo conta com fatores impulsionadores e contracionistas. Por um lado, o fator impulsionador representa os benefícios advindos do acúmulo de conhecimentos, majoritariamente representados pelo acúmulo de experiências devidas às novas tecnologias assimiladas como também às conexões realizadas entre os indivíduos, gerando canais de comunicação. Por outro lado, o fator contracionista é representado pelo custo de absorção de conhecimentos. Assumimos que o custo de absorção de conhecimentos aumenta conforme o país se aproxima da fronteira tecnológica (NARULA, 2004; VERSPAGEN, 1993). 
Nesse sentido, o modelo a ser desenvolvido irá determinar a trajetória do hiato tecnológico em conjunto com a trajetória da capacidade absortiva. A associação desses dois fatores representa a interação que permite desvendar a trajetória do processo de catching-up em países engajados nesse processo de desenvolvimento.

Por fim, como uma forma de simplificar o modelo, suporemos que existem somente dois países no mundo. O Norte, tecnologicamente desenvolvido e, o Sul, tecnologicamente atrasado.

\section{O hiato tecnológico}

Essa seção busca descrever o hiato tecnológico a partir de duas premissas essenciais. Em primeiro lugar, todo o processo de desenvolvimento tecnológico ocorre nos países avançados, ou seja, no Norte. Em segundo lugar, cabe ao Sul se engajar em um processo de desenvolvimento de catching-up para atingir uma convergência na renda. A partir dessas duas premissas, o hiato tecnológico representa o estágio de desenvolvimento obtido pelo Sul frente ao Norte

Representaremos o hiato tecnológico tal como apresentado por Verspagen (1993). Assim, o hiato tecnológico, G, é a relação entre o nível tecnológico do Norte, Tn e o nível Tecnológico do Sul, Ts. Consequentemente, G=Tn/Ts.

Portanto, quanto maior for ' $G$ ', maior será o hiato tecnológico. Quanto mais próximo ' $G$ ' ficar da unidade, menor será o hiato tecnológico. Se em algum momento ' $\mathrm{G}$ ' for menor do que a unidade, o nível tecnológico do Sul será maior do que o nível tecnológico do Norte, representando a quebra do modelo.

Adicionalmente, supomos tal como Narula (2004), Borenztein et al. (1998) e $\mathrm{Xu}$ (2000) que o Norte desenvolve continuamente tecnologias. Neste caso, enquanto o Sul não iniciar o processo de catching-up, a trajetória do hiato deve ter uma taxa de crescimento constante, representada aqui por $\alpha_{0}$.

Mas, se o Sul iniciar o processo de catching-up por meio de algum esforço e atingir a capacidade absortiva mínima necessária, o hiato tecnológico será observado pelo Sul como uma promessa de crescimento futuro (FAGERBERG, 1994). Nesse sentido, quanto maior for o hiato tecnológico, após ser transposto o 
nível mínimo de capacidade absortiva, mais tecnologias estarão disponíveis para serem imitadas/internalizadas pelo Sul.

Porém o processo de internalização das tecnologias não ocorre sem custos, representando determinado engajamento do Sul. Assim, para representar esse custo de internalização, assumimos uma relação inversa dos custos ${ }^{2}$, determinada por $\alpha_{1}$. Assim, quanto maior for $\alpha_{1}$, menor serão os custos. De modo equivalente, quanto menor for $\alpha_{1}$, maiores serão os custos.

Dadas as definições de parâmetros, podemos introduzir a função dinâmica que representa a taxa de crescimento, ou decrescimento, do hiato tecnológico. Portanto:

$$
\hat{G}=\alpha_{0}-\alpha_{1} G \delta
$$

Cabe ressaltar que o símbolo ' $\wedge$ ' acima de determinada variável representa a sua respectiva taxa de crescimento. Dessa forma, o símbolo $\hat{G}$ representa a taxa de crescimento do hiato tecnológico. Nesse sentido, vemos por (2.1) que quando a capacidade absortiva é zero, i.e., $\delta=0$, a taxa de crescimento do hiato tecnológico é igual a $\alpha_{0}$. Isso significa que a falta de capacidade absortiva mínima acaba por implicar em aumentar o hiato tecnológico, ou seja, uma elevação do índice tecnológico do Norte, Tn, e uma estagnação do índice tecnológico do Sul, Ts. Assim, o hiato tecnológico crescerá a uma taxa constante determinada pela taxa de crescimento da fronteira tecnológica.

Se houver algum nível de capacidade absortiva, i.e., $\delta>0$, então a dinâmica do hiato balanceará os seus custos e benefícios para os países do Sul, determinando a convergência tecnológica e da renda entre os países. Para que exista convergência entre as economias, é estritamente necessário que $\delta>\alpha_{0} / \alpha_{1} G$. Esse fato pode ser interpretado como a necessidade de capacidade absortiva mínima para que o

\footnotetext{
${ }^{2}$ É estritamente necessário inserir a inversa dos custos de imitação para que este parâmetro opere com o sinal correto na equação da taxa de crescimento do hiato tecnológico. Como esperamos que uma elevação no custo de imitação acabe por reduzir a taxa de internalização de tecnologias, i.e., aumentar a taxa de crescimento do hiato tecnológico, que é representado por um sinal de negativo em frente ao parâmetro $\alpha_{1}$ de modo a refletir a influência do produto resultante das variáveis do hiato em nível e da capacidade absortiva, é necessário introduzirmos a inversa do custo de imitação de modo a simplificar ao máximo a equação.
} 
processo de catching-up seja executado de forma eficiente (NARULA, 2004; BORENZTEIN et al., 1998; XU, 2000).

Por fim, a equação (2.1) demonstra os dois fatores conectados ao hiato tecnológico. Por um lado, o fator contracionista é demonstrado pela capacidade absortiva mínima para iniciar o processo de catching-up. A equação demonstra que a capacidade absortiva mínima deve ser maior do que o avanço da fronteira tecnológica multiplicada pelos custos imitação das tecnologias ${ }^{3}$. Assim, quanto maior a taxa de crescimento da fronteira tecnológica associada ao custo de imitação da tecnologia, maior deverá ser o investimento do Sul na aquisição da capacidade absortiva mínima.

Por outro lado, o fator impulsionador é determinado pelo tamanho do hiato tecnológico, quanto maior o hiato, menor a capacidade absortiva mínima. Assim, dado o produto entre a taxa de crescimento da fronteira tecnológica e os custos de imitação das tecnologias, quanto maior for o hiato tecnológico, menor será a necessidade de capacidade absortiva mínima para iniciar o processo de catching-up.

Concluindo, o modelo assume que a capacidade absortiva mínima necessária para que se inicie o processo de catching-up está relacionada a dois fatores. O custo de imitação das tecnologias e o hiato tecnológico. Se por um lado o custo de imitação das tecnologias é constante, por outro lado o hiato tecnológico é dinâmico, permitindo que a capacidade absortiva mínima para iniciar o processo de catching-up se reduza conforme o hiato tecnológico aumente.

\section{A capacidade absortiva}

Esta seção tem como objetivo descrever os fatores que influenciam a aquisição de capacidade absortiva, levando em consideração tanto os fatores institucionais quanto do país. Em um primeiro momento cumpre destacar que a assimilação de capacidade absortiva tem uma característica cumulativa, uma vez que o conceito está diretamente relacionado à capacidade cognitiva das pessoas. Assim, assumimos que existe um custo para a absorção de informações associada a determinados benefícios (NARULA, 2004; BARRO; SALA-I-MARTIN, 2003; VERSPAGEN, 1993; COHEN; LEVINTHAL, 1989).

\footnotetext{
${ }^{3}$ Devemos nos lembrar que o parâmetro $\alpha_{1}$ representa a inversa dos custos de imitação das tecnologias.
} 
A capacidade absortiva está associada à parâmetros institucionais que representam um conjunto de elementos particulares de cada país. Neste caso, podemos sintetizá-los como: a saúde dos trabalhadores; a qualidade de ensino das escolas e universidades; o respeito às leis instituídas no país; a forma organizacional da sociedade; a relação entre capital e trabalho; a qualidade dos estimadores sociais, entre outros similares (LALL, 1992). Nesse instante, cabe uma definição conceitual de parâmetros institucionais e aqui seguiremos Edquist e Johson (1997) apud Narula que os definem como (2004): "sets of common habits, routines, established practices, rules, or laws that regulate the interaction between individuals and groups".

Dada a dificuldade em se mensurar os parâmetros institucionais utilizaremos uma escala que compara cada país aos demais. Assim, tomamos como medida uma reta real entre os números 1 a 9 , em que o país que obtiver a classificação 1 conta com parâmetros institucionais melhores do que os outros. De forma similar, o país que obtiver o conceito 9 terá as piores instituições nessa comparação.

Uma forma mais simples de modelar esta relação é inserir o parâmetro institucional como um fator negativamente correlacionado com a taxa de crescimento da capacidade absortiva. Como resultado, obtemos que o país com as melhores condições institucionais obtém uma menor influência negativa sobre a taxa de crescimento da capacidade absortiva.

$\mathrm{Na}$ equação (3.1), consideraremos que o parâmetro ' $\beta_{0}$ ' representa as instituições na taxa de crescimento da capacidade absortiva. Assim os aspectos institucionais influenciam negativamente a taxa de crescimento.

Nesse momento podemos introduzir as características cumulativas do processo de aprendizagem, ou seja, da criação das capacidades absortivas. Elas estão ligadas à procura e aplicação eficiente de tecnologias já desenvolvidas em outros países. Em outras palavras, a capacidade absortiva é conectada ao conceito de hiato tecnológico. Enquanto houver um hiato tecnológico, haverá uma capacidade absortiva que deve representar a capacidade de aprendizado do país, sendo esta influenciada pelo acúmulo de informações e de experiências na internalização de novas tecnologias (NARULA, 2004; VERSPAGEN, 1993; COHEN; LEVINTHAL, 1989; ABRAMOVITZ, 1986). 
Nesse ponto, seguiremos Narula (2004) que correlacionou a capacidade absortiva ao nível de desenvolvimento de cada país, tomando como proxy para o nível de desenvolvimento o hiato tecnológico. Assim, conectamos o pensamento desenvolvido pelo autor com a particularidade do modelo de Verspagen (1993), vinculando o hiato tecnológico à sua capacidade absortiva.

Se por um lado há um efeito cumulativo na capacidade absortiva, por outro lado também existe um efeito de divergência. Observando esse ponto a partir da visão do Sul do hiato tecnológico, esse hiato é tanto uma promessa de crescimento quanto uma necessidade de maior capacidade absortiva para iniciar o processo de catching-up. Nesse sentido, é interessante seguir o desenvolvimento realizado pelo modelo de Verspagen (1993), ao inserir pesos diferenciados para cada efeito.

Nesse momento, introduzimos a equação (3.1), que representa a dinâmica da capacidade absortiva:

$$
\hat{\delta}=-\beta_{0}-\beta_{1} G+\beta_{2} G^{2} \delta
$$

A equação (3.1) deve ser vista como o efeito líquido entre os diversos fatores que influenciam a taxa de crescimento da capacidade absortiva. Observamos que há um efeito negativo proporcionado pelo hiato tecnológico em conjunto com o parâmetro ' $\beta_{1}$ ' e um efeito positivo gerado pelo hiato tecnológico em conjunto com o parâmetro ' $\beta_{2}$ '.

O efeito negativo é derivado do custo de absorção de novas tecnologias. Este custo é definido pelo parâmetro ' $\beta_{1}$ ', supondo que esse parâmetro é constante. Infelizmente não é possível modificar esse parâmetro conforme o hiato tecnológico é reduzido dentro desse modelo matemático. Devido a essa limitação, inserimos essa diferenciação sobre os benefícios advindos do acúmulo de conhecimentos, representado pelo parâmetro ' $\beta_{2}$ '. Essa alteração permite que a equação resultante correspondente à dinâmica da capacidade de absorção seja reduzida conforme o hiato se reduz. Consequentemente, uma redução no hiato tecnológico tende a reduzir mais os benefícios totais proporcionados pelo acúmulo de conhecimentos (i.e., o produto entre o parâmetro ' $\beta_{2}$ ' e o hiato tecnológico elevado ao quadrado) do que propriamente os custos de absorção de conhecimentos.

Finalmente, uma análise mais detida dessa equação demonstra que a capacidade de absorção será positivamente influenciada quanto maior for hiato 
tecnológico. De outra forma, conforme o hiato tecnológico se reduz, i.e., se aproxima de zero, maiores serão os custos e menores serão os benefícios.

Cabe ressaltar um aspecto na equação (3.1) que foi negligenciado até o momento. Na parte correlacionada aos benefícios resultantes da acumulação de novos conhecimentos, também é inserido o nível da capacidade absortiva. A justificativa para este elemento é a incapacidade de iniciar o processo de catchingup se não houver uma capacidade absortiva mínima. Consequentemente, quando $\delta=0$, o Sul não pode extrair qualquer benefício do hiato tecnológico.

Desde que a capacidade absortiva tome valores iguais a zero, haverá uma tendência para que a capacidade absortiva tenda a valores negativos, com o hiato tecnológico crescendo continuamente. Esse fato é observado em alguns países que seguem uma trajetória de divergência no cenário mundial, onde o hiato tecnológico tende a elevar-se em consequência da falta de capacidade absortiva suficiente para iniciar a trajetória de crescimento via catching-up (UNCTAD, 2005).

\section{O ponto de equilíbrio}

Esta seção tem por objetivo investigar a estabilidade do ponto de equilíbrio de longo prazo da iteração dinâmica das equações (2.1) e (3.1) que descrevem um modelo matemático baseado em um processo de catching-up. Conforme descrito nas seções anteriores, esse modelo assumiu a existência de um hiato tecnológico dinâmico com crescimento baseado unicamente no Norte, enquanto ao Sul resta a função de aproveitar o hiato para imitar, tendo em vista um custo não desprezível associado a esse processo. O custo é observado pela necessidade de desenvolvimento de uma capacidade de absorção, influenciada pelo próprio hiato tecnológico.

Igualando as equações (2.1) e (3.1) à zero, de modo a obtermos o seu equilíbrio no estado estacionário, obtemos duas equações como resultado, denominadas de (4.1) e (4.2):

$$
\begin{aligned}
& \delta_{1}=\frac{\alpha_{0}}{\alpha_{1} G} \\
& \delta_{2}=\frac{\beta_{0}+\beta_{1} G}{\beta_{2} G^{2}}
\end{aligned}
$$


Igualando as equações resultantes, (4.1) e (4.2), obteremos a solução para o hiato de equilíbrio, que corresponde a:

$$
G^{*}=\frac{\alpha_{1} \beta_{0}}{\alpha_{0} \beta_{2}-\alpha_{1} \beta_{1}}
$$

Como nessa análise não faz sentido obter um hiato tecnológico negativo (fato associado ao Sul tecnologicamente desenvolvido e o Norte tecnologicamente atrasado), fazemos uma restrição quanto aos parâmetros, para obtermos um hiato positivo. Portanto, por hipótese, $\alpha_{0} \beta_{2}>\alpha_{1} \beta_{1}$.

Inserindo a solução (4.3) na equação (4.1), obtemos a capacidade absortiva de equilíbrio, que corresponde a:

$$
\delta^{*}=\frac{\alpha_{0}}{\alpha_{1} \frac{\alpha_{1} \beta_{0}}{\alpha_{0} \beta_{2}-\alpha_{1} \beta_{1}}}
$$

Nesse momento é interessante descobrirmos como os parâmetros influenciam os valores de equilíbrio do hiato tecnológico e da capacidade absortiva. Portanto, procedemos ao cálculo das derivadas parciais das equações (4.3) e (4.4) com relação aos parâmetros, inserindo os resultados na Tabela 4.1

Tabela 4.1 - Derivadas parciais dos valores de equilíbrio

\begin{tabular}{|c|c|c|}
\hline & $\frac{\partial \delta^{*}}{\partial}$ & $\frac{\partial G^{*}}{\partial}$ \\
\hline$\frac{\partial}{\partial \alpha_{0}}$ & $\frac{2 \alpha_{0} \beta_{2}-\alpha_{1} \beta_{1}}{\alpha_{1}^{2} \beta_{0}}>0$ & $\frac{-\beta_{2} \alpha_{1} \beta_{0}}{\left(\alpha_{0} \beta_{2}-\alpha_{1} \beta_{1}\right)^{2}}<0$ \\
\hline$\frac{\partial}{\partial \alpha_{1}}$ & $-\frac{\alpha_{0} \alpha_{1}^{2} \beta_{1} \beta_{0}+2 \alpha_{1} \beta_{0}}{\left(\alpha_{1}^{2} \beta_{0}\right)^{2}}<0$ & $\frac{\alpha_{0} \beta_{0} \beta_{2}}{\left(\alpha_{0} \beta_{2}-\alpha_{1} \beta_{1}\right)^{2}}>0$ \\
\hline$\frac{\partial}{\partial \beta_{0}}$ & $-\frac{\alpha_{1} \alpha_{0}\left(\alpha_{0} \beta_{2}-\alpha_{1} \beta_{1}\right)}{\left(\alpha_{1}^{2} \beta_{0}\right)^{2}}<0$ & $\frac{\alpha_{1}}{\alpha_{0} \beta_{2}-\alpha_{1} \beta_{1}}>0$ \\
\hline$\frac{\partial}{\partial \beta_{1}}$ & $\frac{-\alpha_{0} \alpha_{1}}{\alpha_{1}^{2} \beta_{0}}<0$ & $\frac{\alpha_{1}^{2} \beta_{0}}{\left(\alpha_{0} \beta_{2}-\alpha_{1} \beta_{1}\right)^{2}}>0$ \\
\hline$\frac{\partial}{\partial \beta_{2}}$ & $\frac{\alpha_{0}{ }^{2}}{\alpha_{1}^{2} \beta_{0}}>0$ & $\frac{-\alpha_{0} \alpha_{1} \beta_{0}}{\left(\alpha_{0} \beta_{2}-\alpha_{1} \beta_{1}\right)^{2}}<0$ \\
\hline
\end{tabular}


Diversos resultados interessantes relativos à elevação ou redução de parâmetros exógenos surgem da análise dos resultados da Tabela 4.1. Em primeiro lugar, variações sobre a taxa de crescimento da fronteira tecnológica influenciada por fatores conectados com o Norte, i.e., uma elevação em ' $\alpha_{0}$ ', gera um efeito líquido positivo para os países do Sul engajados em um processo de catching-up. Assim a aceleração da expansão da fronteira tecnológica incrementa a capacidade absortiva dos países do Sul que já ultrapassaram o nível mínimo de capacidade absortiva, reduzindo hiato tecnológico entre os países no equilíbrio de longo prazo. Dessa forma, uma elevação sobre ' $\alpha_{0}$ ' pode representar, em um primeiro momento, a elevação do hiato de equilíbrio, mas esta elevação sobre o hiato influencia positivamente a capacidade absortiva ao expandir a quantidade de tecnologias a serem imitadas. Ao final desse processo dinâmico o resultado é um hiato de equilíbrio menor e uma capacidade absortiva maior.

De maneira inversa, uma redução dos custos de imitação, representado por ' $\alpha_{1}$ ', acaba por influenciar negativamente o hiato tecnológico e positivamente a capacidade absortiva. Como o custo de imitação é uma variável que influencia tanto a capacidade absortiva como o hiato tecnológico, elevações nesse parâmetro resultam na necessidade de maior investimento na capacidade absortiva. Nesse sentido, uma redução dos custos de imitação deve ser visualizada como uma redução na dificuldade em se imitar, consequentemente em uma redução da preocupação por parte dos empreendedores e do governo em alcançar maiores capacidades absortivas, o que gera um hiato tecnológico maior do que o observado anteriormente. Portanto, podemos inserir a discussão de Barro e Sala-i-Martin (2003), representando o fato de que quanto mais próximos à fronteira de conhecimentos, maior será o custo de imitação. Consequentemente, quanto maior for o custo de imitação, maior será a capacidade absortiva necessária e menor será o hiato tecnológico.

Quando o parâmetro institucional, ' $\beta_{0}$ ', sofre uma alta, ou seja, ocorre uma piora nas instituições do país, o hiato tecnológico sofrerá uma elevação e a capacidade absortiva sofrerá uma redução. Nesse sentido, melhoras nos parâmetros institucionais ligados a uma estratégia de catching-up reforça a capacidade absortiva e reduz o hiato de equilíbrio. Como destacado por Xu (2000), esta é uma estratégia que tem efeitos favoráveis (XU, 2000). 
Uma elevação nos custos de absorção, ' $\beta_{1}$ ', resultam em um hiato tecnológico maior e uma capacidade absortiva menor. De fato, a elevação no custo de absorção deve estar ligada a redução da capacidade absortiva, uma vez que o tamanho do hiato tecnológico terá menor influência sobre a aquisição de tal capacidade.

Por fim, uma elevação proveniente dos benefícios advindos do acúmulo de conhecimentos, ' $\beta_{2}$ ', resultará em uma elevação da capacidade absortiva e subsequente redução do hiato tecnológico. Isso está ligado ao fato de que agora, com o mesmo nível do hiato tecnológico, mas com um ' $\beta_{2}$ ' maior, o Sul pode aproveitar mais as possibilidades abertas pelo hiato, de modo a internalizar com maior eficiência as tecnologias.

Resta-nos, agora, determinar quais são as condições para que este equilíbrio seja estável. Montando a matriz jacobiana ${ }^{4}$ e observando o seu traço e respectivo determinante, encontra-se:

$$
\begin{aligned}
& J=\left(\begin{array}{cc}
-\alpha_{1} \delta & -\alpha_{1} G \\
-\beta_{1}+2 \beta_{2} G & \beta_{2} G^{2}
\end{array}\right) \\
& \operatorname{det}=\alpha_{1} \beta_{2} G^{2} \delta-\alpha_{1} G \beta_{1} \\
& \operatorname{tr}=-\alpha_{1} \delta+\beta_{2} G^{2}
\end{aligned}
$$

As condições para a estabilidade do ponto requerem que o determinante seja positivo e que o traço seja negativo. Assim, emergem da resolução da matriz jacobiana as seguintes condições de estabilidade:

$$
\begin{gathered}
\delta>\frac{\beta_{2} G^{2}}{\alpha_{1}} \\
G>\frac{\beta_{1}}{\beta_{2} \delta}
\end{gathered}
$$

A condição (4.5) pode ser vista como o nível mínimo de capacidade absortiva para que o Sul inicie o processo de catching-up. Portanto, esse nível mínimo está correlacionado com o nível do hiato tecnológico. Quanto maior o hiato, maior será a capacidade absortiva mínima requerida para que o processo de catching-up seja estável no longo prazo. Da mesma forma, quanto maior for o

\footnotetext{
${ }^{4}$ Para detalhes sobre o cálculo do Jacobiano, do determinante da matriz resultante, seu traço e das condições de estabilidade, veja Simon e Blume (2004), Viana (2008), Gandolfo (1997) e Chiang e Wainwright (2006).
} 
custo proveniente da imitação das tecnologias, maior deverá ser a capacidade absortiva inicial para impulsionar o processo de catching-up. Por fim, uma elevação dos benefícios advindos do acúmulo de conhecimentos implica na necessidade de uma maior capacidade absortiva mínima para que haja estabilidade no sistema.

Já a condição (4.6) demonstra que o hiato tecnológico deve ser maior do que a razão entre o custo de absorção de conhecimentos, ' $\beta_{1}$ ', e o produto entre os benefícios advindos do acúmulo de conhecimentos e a capacidade absortiva, ' $\beta_{2} \delta$ '. Assim, quanto maior for a capacidade absortiva ou os benefícios provenientes do acúmulo de conhecimentos, menor deve ser o hiato tecnológico para que haja um ponto com equilíbrio estável. Da mesma forma, quanto maior for o custo de obtenção de conhecimentos, maior deverá ser o hiato. Esse fato reflete a relação de custos que existe no processo de catching-up. Assim, deve existir um nível mínimo de hiato entre os países para que o menos desenvolvidos consigam iniciar a sua trajetória de desenvolvimento via catching-up. Este nível mínimo de hiato tecnológico é relacionado com os custos e benefícios provenientes da acumulação de capacidade absortiva bem como dos benefícios e custos resultantes da introdução de novas tecnologias, como demonstrado na equação (4.6).

Desde que estas duas condições determinadas por (4.5) e (4.6) sejam satisfeitas, pode-se afirmar que o ponto de equilíbrio resultante nas duas equações, (4.3) e (4.4), são estáveis.

O presente modelo incrementa as conclusões dos artigos de Verspagen (1993), Narula (2004) e Criscuolo e Narula (2002). Enquanto nos artigos citados os autores afirmam ser necessária uma capacidade absortiva mínima sem a definir explicitamente. Entretanto, o modelo desenvolvido tem como um de seus principais resultados um nível mínimo de capacidade absortiva que é obtida como condição necessária para a estabilidade.

Como observado na resolução acima, há um ponto de equilíbrio estável demonstrando que a economia do Sul chegará a um ponto em que o seu crescimento relativo à economia do Norte será constante. Essa estabilização ocorre na fase de pré-compartilhamento da fronteira tecnológica (Narula, 2004). Nesse sentido, o equilíbrio estável garante ao país tecnologicamente atrasado a possibilidade de crescimento e de desenvolvimento até determinado limite. Esse 
limite representa o esgotamento da estratégia de crescimento através do processo de catching-up. A presença deste limite revela a necessidade de mudar a estratégia de desenvolvimento buscando ultrapassar a fronteira tecnológica por meio do processo inovativo. Portanto, ao obter o equilíbrio, demonstrou-se que a estratégia de desenvolvimento via catching-up aliada ao desenvolvimento da capacidade absortiva é coerente com uma estratégia para obter a convergência da renda, contudo esta deve ser complementada no futuro com o desenvolvimento das instituições do país, não mais voltadas para o aprendizado e sim para a inovação.

Segundo Viotti (2000), o equilíbrio da estratégia de catching-up representa o sucesso do país no desenvolvimento de seu Sistema Nacional de Aprendizado (SNA). Contudo, o equilíbrio estável obtido no modelo desta seção demonstra a necessidade de evolução do país, de uma estrutura correlacionada de um SNA bem-sucedido para um SNI, que se tornará a base de inovação e subsequente crescimento do país. Nesse instante, o modelo desenvolvido aqui não se justificaria mais, uma vez que o país fará parte das nações que compartilham a fronteira tecnológica, ou seja, o Norte.

\section{Análise gráfica}

Esta seção tem por objetivo demonstrar o diagrama de fase do modelo com as respectivas curvas de demarcação ${ }^{5}$. O primeiro passo já foi dado ao obtermos as soluções de equilíbrio determinadas por (4.3) e (4.4). Determinado o ponto de equilíbrio e sua estabilidade, o diagrama de fase permite realizar uma análise a partir do ponto de equilíbrio e de possíveis novas trajetórias a partir da análise da figura.

As curvas de demarcação representam os pontos do diagrama de fase nos quais as taxas de crescimento das variáveis são iguais a zero. Assim, o nosso primeiro objetivo será encontrar as curvas de demarcação, as quais chamaremos de $\hat{\delta}=0$ para a equação (4.4) e $\hat{G}=0$ para a equação (4.3).

Para tanto, devemos averiguar qual é a inclinação de cada curva de demarcação por meio das seguintes derivadas:

\footnotetext{
${ }^{5}$ Uma boa introdução a resolução de equações dinâmicas lineares é dada por Chiang e Wainwright (2006), cap. 19.
} 


$$
\begin{aligned}
& \frac{\partial \delta_{1}}{\partial G}=-\frac{\alpha_{0} \alpha_{1}}{\left(\alpha_{1} G\right)^{2}}<0 \\
& \frac{\partial \delta_{2}}{\partial G}=\frac{-2 \beta_{2} \beta_{0}-\beta_{2} \beta_{1} G^{2}}{\left(\beta_{2} G^{2}\right)^{2}}<0
\end{aligned}
$$

Via (5.1) e (5.2), sabemos que as inclinações das respectivas curvas de demarcação são negativas. Agora, resta saber qual é o formato da curva, uma vez que não é linear, pode ter a caracterização de um máximo, com uma velocidade, i.e., com uma segunda derivada negativa, ou o formato de um mínimo, com uma velocidade positiva. Investigando estes fatos, obtemos:

$$
\begin{aligned}
& \frac{\partial^{2} \delta_{1}}{\partial G^{2}}=\frac{2 \alpha_{0} \alpha_{1}^{3} G}{\left(\alpha_{1} G\right)^{4}}>0 \\
& \frac{\partial^{2} \delta_{2}}{\partial G^{2}}=\frac{2\left(3 \beta_{0}+\beta_{1} G\right)}{\beta_{2} G^{4}}>0
\end{aligned}
$$

A equação (5.3) demonstra que a curva de demarcação $\hat{G}=0$ é representada por uma curva com um mínimo. Da mesma forma, a equação (5.4) demonstra que a curva de demarcação de $\hat{\delta}=0$ é representada por uma curva negativamente inclinada.

Sabendo a inclinação das respectivas curvas de demarcação e a sua forma, podemos esboçar um diagrama de fase. As curvas de demarcação devem se cruzar somente uma vez no primeiro quadrante. Assim, resta saber qual curva é mais inclinada, se $\hat{\delta}=0$ ou $\hat{G}=0$. Aqui suporemos que $\alpha_{1} \beta_{2} G<3 \beta_{0} \alpha_{1}+\beta_{1} \alpha_{1} G$, ou seja, que o produto $3 \beta_{0} \alpha_{1}$ é grande o suficiente para tornar o lado direito maior que o esquerdo. Feita tal suposição, encontramos que a curva de demarcação correspondente à $\hat{\delta}=0$ tem uma maior inclinação em relação à $\hat{G}=0$, devido a maior velocidade de decrescimento que ocorre devido a suposição realizada previamente. Essa suposição gera a formato visualizado na figura 5.1. 


\section{Figura 5.1}

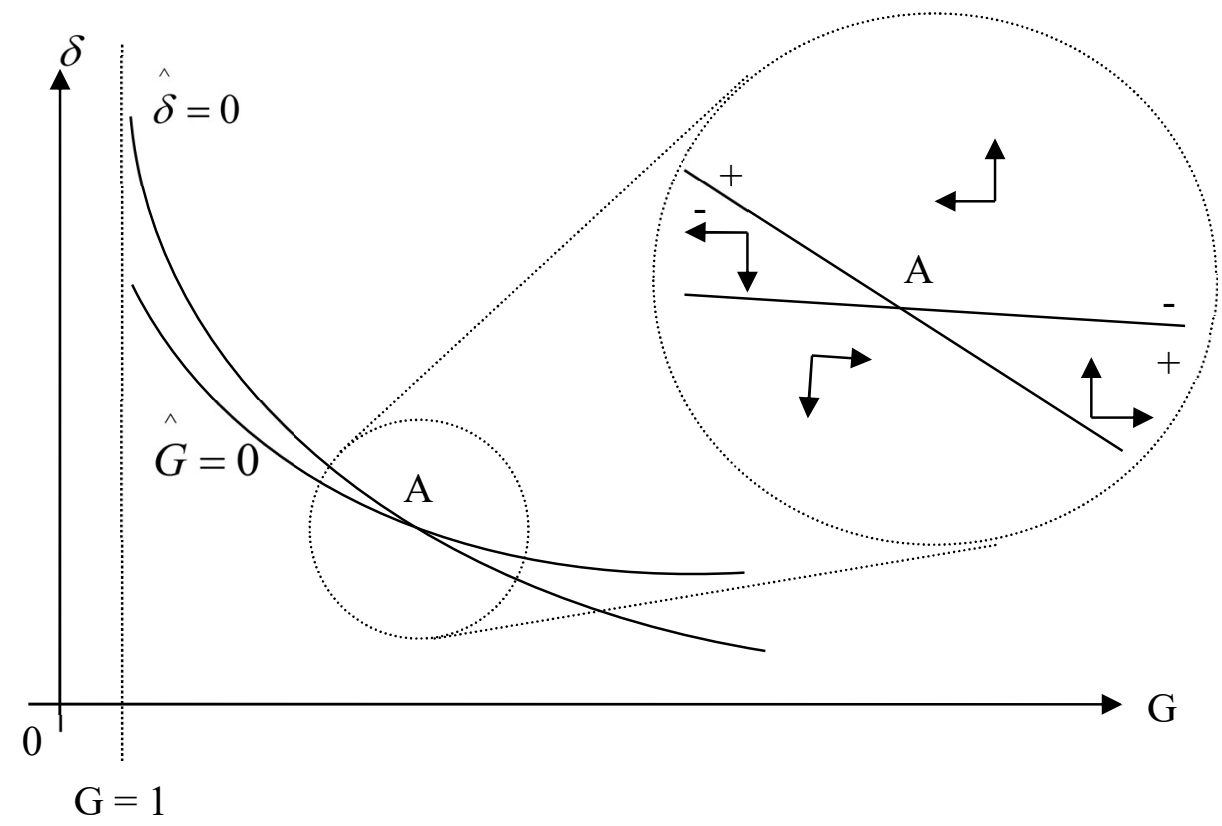

As setas direcionais foram obtidas com o auxílio das derivadas que demonstram a variação entre a taxa de crescimento da variável com a variável em nível, ou seja:

$$
\begin{aligned}
& \frac{\partial \hat{G}}{\partial G}=-\alpha_{1} \delta<0 \\
& \frac{\partial \hat{\delta}}{\partial \delta}=\beta_{2} G^{2}>0
\end{aligned}
$$

O conjunto de equações (5.5) e (5.6) ratificam o sentido de crescimento e decrescimento das setas direcionais e, consequentemente, as linhas de fluxo, não traçadas na figura. Desde que a curva de demarcação corresponda a uma taxa de crescimento igual a zero, se traçarmos uma reta vertical que passe pela curva de demarcação $\hat{\delta}=0$ (como uma forma de demonstrar que mantemos o hiato constante, tal como realizado durante a solução da derivada (5.6)), saberemos que os pontos acima da curva de demarcação e, portanto, pontos que tem um maior nível de capacidade absortiva, estão ligados a uma taxa de crescimento positiva, como demonstrado pela equação (5.6). Nesse sentido, a figura 5.1 demonstra, em sua ampliação, que pontos acima da curva de demarcação $\hat{\delta}=0$ têm o sinal positivo e uma seta direcional que aponta para cima, representando um 
crescimento da capacidade absortiva. Da mesma forma, pontos que estão abaixo da curva de demarcação recebem o sinal negativo e setas direcionais que apontam para baixo, o sentido de decrescimento da capacidade absortiva.

Traçando-se uma reta horizontal que passe pela curva de demarcação $G=0$ de forma a manter a capacidade absortiva constante, pela derivada obtida em (5.5), o lado da reta que tiver mais hiato, i.e., o lado direito da reta, terá um sinal negativo, enquanto o lado esquerdo, que tem um hiato menor, terá o sinal positivo, como demonstrado pela figura 5.1. As setas direcionais seguem os sinais, assim, à direita da curva de demarcação $\hat{G}=0$ encontramos o sinal de negativo e, dessa forma, uma seta direcional que indica o decrescimento do hiato, ou seja, uma seta apontando para a esquerda. Já a direita da curva de demarcação, encontramos o sinal de positivo e a seta direcional apontando para a direita, no sentido de crescimento do hiato tecnológico.

\section{Conclusões}

A principal questão levantada pela literatura sobre hiato tecnológico é, justamente, em que medida o hiato é um fator positivo ou negativo para o crescimento das economias que estão afastadas da fronteira tecnológica. Nesse contexto, a capacidade de cada país em iniciar uma trajetória bem-sucedida de catching-up, aproveitando o potencial de imitação associado ao hiato, é determinada pelas capacidades de aprender, absorver e adaptar a tecnologia importada. Partindo-se do pressuposto de que a tecnologia não é um bem público, uma vez que há custos em sua internalização representado tanto pelos custos de imitação quanto pelo custo de se aquirir as capacidades necessárias para absorver a tecnologia, as políticas e instituições públicas são cruciais à medida que a acumulação de conhecimentos realizados pelas pessoas, firmas e países é um processo cumulativo. Consequentemente pode surgir tanto círculos viciosos de atraso tecnológico e baixo crescimento quanto um círculo virtuoso de crescimento via processo de catching-up. Nesse sentido, se ocorrer um processo de pathdependency em determinados setores com capacidades menos dinâmicas, essa trajetória deve ser rompida com políticas públicas ativas, direcionadas às instituições públicas. 
O modelo proposto mostra que a trajetória do catching-up pode permitir certa convergência, mas não a ultrapassagem. Neste sentido, o Sul nunca consegue ultrapassar o Norte, uma vez que a estratégia do modelo se baseia unicamente na imitação/internalização de tecnologias já desenvolvidas. Portanto, uma trajetória de crescimento de longo prazo também deve incluir a necessidade do desenvolvimento do setor de pesquisa e desenvolvimento, ocasionando a quebra do paradigma e subsequente início do ciclo de crescimento.

O principal ponto de diferenciação entre o modelo apresentado na seção quatro e a literatura sobre o hiato tecnológico é a introdução da capacidade absortiva como uma variável endógena. Como essa variável foi introduzida de modo a ser influenciada pelo hiato tecnológico, emerge do modelo como condição de estabilidade a necessidade de uma capacidade absortiva mínima. Se, por algum motivo, o Sul não obtiver a capacidade absortiva mínima, o seu desenvolvimento por meio da estratégia de catching-up estará fadado ao fracasso, uma vez que a trajetória perseguida pelo país deve ser instável de forma a não podermos obter maiores informações a seu respeito. Contudo, se o Sul obtiver a capacidade absortiva mínima, o modelo torna-se estável, demonstrando todo o potencial da estratégia de crescimento baseada no catching-up além de corroborar a evidência empírica apresentada pelos países da OCDE que se engajaram na estratégia de catching-up. A literatura demonstra que a intensificação da sua capacidade absortiva por meio de políticas públicas voltadas à melhoria educacional e institucional foi um dos segredos da estratégia de catching-up.

O modelo mostra que existem duas capacidades absortivas mínimas. A primeira é estritamente necessária para que haja um equilíbrio no modelo, enquanto a segunda é necessária para que haja convergência.

O equilíbrio com um hiato tecnológico estável pode ser interpretado como um esgotamento da estratégia de catching-up, implicando na necessidade de intensificação das políticas governamentais que estejam voltadas à inovação. Para fechar o hiato, o país deve iniciar outra fase de desenvolvimento, não mais baseada no aprendizado nem na introdução de novas tecnologias, e sim na inovação. 


\section{Referências}

ABRAMOVITZ, M. Catching Up, Forging Ahead, and Falling Behind. Journal of Economic History, v. 46, n. 2, p. 386-406, 1986.

ARROW, J. K. The economic implications of learning-by-doing. Review of Economic Studies, v. 29, p. 155-73, 1962.

BARRO, R. J.; SALA-i-MARTIN, X. Economic Growth. Cambridge: The MIT Press, 2003.

BAUMOL, W. J. Productivity Growth, Convergence, and Welfare: What the Long-run Data Show. American Economy Review, v. 76, n. 5, p. 1072-1085, 1986.

BAUMOL, W. J.; BLACKMAN, S. A. B.; WOLFF, E. N. Productivity and American leadership. Cambridge: The MIT Press, 1989.

BORENSZTEIN, E; GREGORIO, J.; LEE, J.W. How Does Foreign Direct Investment Affect Economic Growth. Journal of International Economics, v. 45, n. 1, p. 115-135, 1998.

CHIANG, A. C.; WAINWRIGHT, K. Matemática para Economistas. São Paulo: Elsevier, 2006.

COHEN, W. M.; LEVINTHAL, D. Innovation and learning: The two faces of R\&D. The Economic Journal, v. 99, p. 569-596, 1989.

CRISCUOLO, P.; NARULA, R. A novel approach to national technological accumulation and absorptive capacity: Aggregating Cohen and Levinthal. MERIT Research Memorandum, p. 2002-2016, 2002.

DAHLAN, C. J.; NELSON R. Social Absorption Capability, National Systems and Economic Development. In: HO KOO. Social capability and long-term economic growth. Londres: St. Martin's Press, 1995.

DE LONG, J. B. Productivity Growth, Convergence, and Welfare: Comment, American Economic Review, v. 78, n. 5, p. 1138-1154, 1988.

FABERBERG, J. Why Growth Rates Differ. In: DOSI, G.; FREEMAN, C.; SILVERBERG, G.; SOETE, L. (Eds.). Technical change and economic theory. Londres: Cambridge University Press, 1988b. 
FAGERBERG, J. A Technology Gap Approach to Why Growth Rates Differ, Research Policy, v. 16, n. 2-4, p. 87-99, 1987.

FAGERBERG, J. International competitiveness. Economic Journal, v. 98, p. 355374, 1988a.

FAGERBERG, J. Technology and International Differences in Growth Rates. Journal of Economic Literature, v. 32, n. 2, p. 1147-1175, 1994.

FREEMAN, C. A hard landing for the 'New Economy'? Information technology and the United States National System of Innovation. Structural Change and Economic Dynamics, v. 12, n. 2, p. 115-139, 2001.

FREEMAN, C. The National System of Innovation in Historical Perspective. Cambridge Journal of Economics, v. 19, n. 1, p. 5-24, 1995.

GANDOLFO, G. Economic dynamics. Berlin-Heidelberg-New York, 1997.

LALL, S. Technological capabilities and industrialization. World Development, v. 2, n. 2, p. 165-186, 1992.

LÉON-LEDESMA, M. A. Accumulation, Innovation and catching-up: an extended cumulative growth model. Cambridge Journal of Economics, v. 26, n. 2, p. 201-216, 2002.

MADDISON, A. Growth and Slowdown in Advanced Capitalist Economies. Journal of Economy Literature, v. 25, n. 2, p. 649-698, 1987.

McCOMBIE, J. S. L.; THIRLWALL, A. P. Economic Growth and Balance of Payments Constraint. New York: St Martin's Press, 1994.

NARULA, R. Understanding Absorptive Capacities in an Innovation Systems Context: Consequences for Economic and Employment Growth. DRUID Working Paper, n. 04-02, 2004.

NARULA, R.; CRISCUOLO, P. A novel approach to national technological accumulation and absorptive capacity: Aggregating Cohen and Levinthal. MERIT Research Memorandum, p. 2002-2018, 2002.

NARULA, R.; MARIN, A. FDI spillovers, absorptive capacities and human capital development: evidence from Argentina. MERIT Infonomics Research Memorandum, 2003. 
NELSON, R.; PHELPS, E. Investments in human, technological diffusion and economic growth. American Economic Review, v. 61, p. 69-75, 1966.

NELSON, R.; WINTER, S. An Evolutionary Theory of Economic Change. Cambridge: Harvard University Press, 1982.

PORCILE G.; CIMOLI, M. Tecnología, Diversificación productiva y Crecimiento: Um Modelo Estructuralista. Economia e Sociedade, v. 16, n. 3, 2007.

PORCILE, G.; DUTRA, M. V.; MEIRELlES, A. J. A. Technology gap, real wages, and learning in a balance-of-payments constrained growth model. Journal of Post Keynesian Economics, v. 29, n. 3, p. 473-500, 2007.

SHONE, R. Economic dynamics: phase diagrams and their economic application. Cambridge: Cambridge University Press, 1997.

SIMON, C. P.; BLUME, L. Matemática para Economistas. Porto Alegre: Bookman, 2004.

TARGETTI, F.; FOTI, A. Growth and productivity: a model of cumulative growth and catching up. Cambridge Journal of Economics, v. 21, n. 1, p. 27-43, 1997.

UNCTAD. Issues Paper on Bridging the technology gap. Morocco, 2005.

VERSPAGEN, B. Uneven growth between interdependent economies. London: Avebury, 1993.

VIANA, R. Introdução a Dinâmica Econômica Não-Linear e Caos. Curitiba: UFPR, 2008.

VIOTTI, E. B. National Learning Systems A new approach on technical change in late industrializing economies and evidences from the cases of Brazil and South Korea. In: 4th International Conference on Technology Policy and Innovation Learning and Knowledge Networks for Development. Curitiba, p. 28-31, 2000.

$\mathrm{XU}$, B. Multinational enterprises, technology diffusion, and host country productivity growth. Journal of Development Economics, v. 62, p. 477-493, 2000. 